\title{
On a 3D Initial-Boundary Value Problem for Determining the Dynamics of Impurities Concentration in a Horizontal Layered Fine- Pore Medium
}

\author{
Sharif E. Guseynov \\ Faculty of Science and Engineering \\ Institute of Fundamental Science and \\ Innovative Technologies \\ Liepaja University \\ Liepaja, Latvia \\ "Entelgine" \\ Research \& Advisory Co., Ltd. \\ Riga, Latvia \\ sh.e.guseinov@inbox.lv
}

\section{Ruslans Aleksejevs}

Faculty of Mechanics and Mathematics

Lomonosov Moscow State University

Moscow, Russia

aleksejevs.ruslans@gmail.com

\author{
Jekaterina V. Aleksejeva \\ Institute of Fundamental Science and \\ Innovative Technologies \\ Liepaja University \\ Riga Secondary School 34 \\ Riga, Latvia \\ jekaterina.v.aleksejeva@gmail.com
}

\begin{abstract}
In the present paper, we propose an analytical approach for solving the 3D unsteady-state boundary-value problem for the second-order parabolic equation with the second and third types boundary conditions in two-layer rectangular parallelepipedic domain.
\end{abstract}

Keywords - unsteady-state diffusion equation, initialboundary value problem, Robin boundary condition, analytical method.

\section{INTRODUCTION}

In the present paper, we propose an analytical approach for solving the 3D unsteady-state boundary-value problem for the second-order parabolic equation with the third type boundary conditions in two-layer rectangular parallelepipedic domain. Such type problems arise in particular at study of metal concentration dynamics in the peat blocks (for instance, see [1]-[5] and respective references given in these). Mathematical statement of the considered problem is taken from the article [1], where the problem was solved by combination of the two approaches: firstly, the averaging method in the vertical direction (i.e. in height) and two horizontal directions (i.e. in width and in length), and, then, the obtained 2D problems have been solved by the standard/classical analytical methods. As opposed to the combinational approach suggested in [1], in the present paper, we do not use approximation methods at all (basically, result of application of the averaging method always is approximate).

\section{Mathematical FORMUlation OF PROBLEM}

Denote by $\Omega_{i x} \quad i$-th $(i=1,2)$ layer of two-layer peat block, which has shape of rectangular parallelepiped:

$$
\begin{aligned}
& \Omega_{i x} \stackrel{\text { def }}{\equiv}\left\{x=\left(x_{1}, x_{2}, x_{3}\right) \in \mathrm{i}^{3} \mid x_{j} \in\left[0, L_{j}\right], j=1,2 ;\right. \\
& \left.(i-1) H_{1} \leq x_{3} \leq(i-1)\left(L_{3}-H_{1}\right)+H_{1}\right\}, i=1,2 .
\end{aligned}
$$

Now we formulate a mathematical model describing the dynamics of metal concentration in a two-layer peat block: it is required to find functions $c_{i}(x, t): \Omega_{i x} \times\left[0, t_{E N D}\right] \rightarrow \mathrm{i}^{1},(i=1,2)$, which satisfy

- diffusion equations with sources

$$
\begin{aligned}
& \frac{\partial c_{i}(x, t)}{\partial t}=\sum_{j=1}^{3} D_{i j} \frac{\partial^{2} c_{i}(x, t)}{\partial x_{j}^{2}}+f_{i}(x, t), \\
& (x, t) \in \operatorname{int} \Omega_{i x} \times\left(0, t_{E N D}\right],(i=1,2) ;
\end{aligned}
$$

- $\quad$ initial conditions

$\left.c_{i}(x, t)\right|_{t=0^{+}}=c_{i 0}(x), x \in \Omega_{i x},(i=1,2) ;$

- the following boundary conditions given:

- $\quad$ at the trailing wall in the form of von Neumann condition

$$
\begin{aligned}
& \left.\frac{\partial c_{i}(x, t)}{\partial x_{1}}\right|_{x_{1}=0^{+}}=c_{i 1}\left(x_{2}, x_{3}, t\right), \\
& \left(x /\left\{x_{1}\right\}, t\right) \in \Omega_{i x /\left\{x_{1}\right\}} \times\left[0, t_{E N D}\right],(i=1,2) ;
\end{aligned}
$$

- $\quad$ at the front wall in the form of Robin condition

$\left.\left[D_{i 1} \frac{\partial c_{i}(x, t)}{\partial x_{1}}+\lambda_{i 1} c_{i}(x, t)\right]\right|_{x_{1}=L_{1}^{-}}=a_{i 1}\left(x_{2}, x_{3}, t\right)$,

$\left(x /\left\{x_{1}\right\}, t\right) \in \Omega_{i x /\left\{x_{1}\right\}} \times\left[0, t_{E N D}\right],(i=1,2) ;$

- at the left-side wall in the form of von Neumann condition

$$
\begin{aligned}
& \left.\frac{\partial c_{i}(x, t)}{\partial x_{2}}\right|_{x_{2}=0^{+}}=c_{i 2}\left(x_{1}, x_{3}, t\right), \\
& \left(x /\left\{x_{2}\right\}, t\right) \in \Omega_{i x /\left\{x_{2}\right\}} \times\left[0, t_{E N D}\right],(i=1,2)
\end{aligned}
$$


- at the right-side wall in the form of Robin condition

$$
\begin{aligned}
& {\left.\left[D_{i 2} \frac{\partial c_{i}(x, t)}{\partial x_{2}}+\lambda_{i 2} c_{i}(x, t)\right]\right|_{x_{2}=L_{2}^{-}}=a_{i 2}\left(x_{1}, x_{3}, t\right),} \\
& \left(x /\left\{x_{2}\right\}, t\right) \in \Omega_{i x /\left\{x_{2}\right\}} \times\left[0, t_{E N D}\right],(i=1,2)
\end{aligned}
$$

- $\quad$ at the lower ( by $i=1$ ) and the upper (by $i=2$ ) bases in the form of Robin condition

$$
\begin{aligned}
& {\left.\left[D_{i 3} \frac{\partial c_{i}(x, t)}{\partial x_{3}}+(2 i-3) \lambda_{i 3} c_{i}(x, t)\right]\right|_{x_{3}=(i-1)^{+} L_{3}^{-}}=a_{i 3}\left(x_{1}, x_{2}, t\right),} \\
& \left(x /\left\{x_{3}\right\}, t\right) \in \Omega_{i x /\left\{x_{3}\right\}} \times\left[0, t_{E N D}\right],(i=1,2) ;
\end{aligned}
$$

- matching conditions given at the bedding interface [6]

$$
\begin{aligned}
& \left.\left((i-1)\left(D_{13}-1\right)+1\right) \frac{\partial^{i-1} c_{1}(x, t)}{\partial x_{3}}\right|_{x_{3}=H_{1}^{-}} \\
& =\left.\left((i-1)\left(D_{23}-1\right)+1\right) \frac{\partial^{i-1} c_{2}(x, t)}{\partial x_{3}}\right|_{x_{3}=H_{1}^{+}}, \\
& \left(x /\left\{x_{3}\right\}, t\right) \in \Omega_{i x /\left\{x_{3}\right\}} \times\left[0, t_{E N D}\right],(i=1,2) ;
\end{aligned}
$$

- all 12 consistency conditions linking the initial and boundary functions from the constraints (2)

In the mathematical model (1)(8) it is assumed that all numerical parameters $L_{i}>0(i=\overline{1,3}), \quad H_{i}>0(i=1,2)$, $D_{i j}>0(i=1,2 ; j=\overline{1,3})$,

$\lambda_{i j}>0(i=1,2 ; j=\overline{1,3})$ $t_{E N D}>0$, and all functions except functions $c_{1}(x, t)$ and $c_{2}(x, t)$, which stand for the desired metal concentrations, respectively, in the first and second layers of the peat block, are a priori given.

Remark 1. If in the mathematical model (1)(8) we assume that: (a) $c_{i 1}(\mathrm{~g})=c_{i 2}(\mathrm{~g}) \equiv 0,(i=1,2)$, (b) boundary functions $a_{i j}(\mathrm{~g}),(i=1,2 ; j=\overline{1,3})$ do not depend on time $t$, then the model (1)(8) will completely coincide with the mathematical model (1.1) from [1], in which the physical interpretations of all the initial data - numerical parameters and functions are exhaustively described. Therefore, in this paper we will not describe the physical meaning of the initial numerical parameters and functions of the model (1)(8): they have the same meaning as in [1]. End of Remark 1.

The mathematical model (1)(8) can be solved by two different approaches. The first approach is a more universal approach for solving wide classes of initial-boundary value problems in layered regions with layers, whose physical, chemical, etc. characteristics are different. The main idea of the first approach is to reduce the mathematical model (1)(8) to the Fredholm integral equation of the first kind, and the subsequent procedure for applying Tikhonov's regularization method to the obtained integral equation
[7], [8]. In view of the strict requirements for the maximum volume of articles, in this paper we will not consider the necessary procedures for performing the first approach. The essence of the second approach consists of applying method of separation of variables and constructing the Green's function (for instance, see [6] that is one of the best mathematical textbooks ever written). This method of solving initial problems (a Cauchy problem, when the region in which the process is studied is an unbounded region), boundary-value problems (in the case when the steady-state process is studied, or the process is studied at a time sufficiently far from the initial moment of the process) and initial-boundary value problems is a more "traditional" approach in the sense that this technique is, firstly, thoroughly studied in almost all courses of equations of mathematical physics and/or partial differential equations, and, secondly, is widely used in the study of various kinds of mathematical models described in the language of initial, boundary and initial-boundary problems for partial differential equations, in particular, for hyperbolic, parabolic and elliptic types of differential equations. In this paper, the second approach is chosen as the analytical method for solving the mathematical model (1)(8) - the method of separation of variables and construction of the corresponding Green's function.

\section{APPLICATION OF THE METHOD OF} SEPARATION OF VARIABLES, AND CONSTRUCTION OF THE CORRESPONDING GREEN'S FUNCTIONS

So, let us consider the initial-boundary value problem (1)(8), and try to find its solution by applying the method of separation of variables. To do this, we first formulate, as is customary in the method of separation of variables [6], two auxiliary boundary-value problems - the problem AP1 and the problem AP2, in each of which the equation is homogeneous.

\section{A. Formulation of two auxiliary boundary-value problems}

Auxiliary problem AP1. It is required to find the function $0 \not c_{1}(x, t): \Omega_{1 x} \times\left[0, t_{E N D}\right] \rightarrow \mathrm{i}^{1}$ that satisfies:

- homogeneous equation

$$
\begin{aligned}
& \frac{\partial c_{1}(x, t)}{\partial t}=\sum_{j=1}^{3} D_{1 j} \frac{\partial^{2} c_{1}(x, t)}{\partial x_{j}^{2}}, \\
& (x, t) \in \operatorname{int} \Omega_{1 x} \times\left(0, t_{E N D}\right],
\end{aligned}
$$

- heterogeneous initial condition

$$
\left.c_{1}(x, t)\right|_{t=0^{+}}=c_{10}(x), x \in \Omega_{1 x},
$$

- $\quad$ homogeneous boundary conditions

$$
\begin{aligned}
& \left.\frac{\partial c_{1}(x, t)}{\partial x_{1}}\right|_{x_{1}=0^{+}}=0,\left(x /\left\{x_{1}\right\}, t\right) \in \Omega_{1 x /\left\{x_{1}\right\}} \times\left[0, t_{E N D}\right], \\
& \left.D_{11} \frac{\partial c_{1}(x, t)}{\partial x_{1}}\right|_{x_{1}=L_{1}^{-}}+\left.\lambda_{11} c_{1}(x, t)\right|_{x_{1}=L_{1}^{-}}=0
\end{aligned}
$$$$
\left(x /\left\{x_{1}\right\}, t\right) \in \Omega_{1 x /\left\{x_{1}\right\}} \times\left[0, t_{E N D}\right],
$$ 
$\left.\frac{\partial c_{1}(x, t)}{\partial x_{2}}\right|_{x_{2}=0^{+}}=0,\left(x /\left\{x_{2}\right\}, t\right) \in \Omega_{1 x /\left\{x_{2}\right\}} \times\left[0, t_{E N D}\right]$

$\left.D_{12} \frac{\partial c_{1}(x, t)}{\partial x_{2}}\right|_{x_{2}=L_{2}^{-}}+\left.\lambda_{12} c_{1}(x, t)\right|_{x_{2}=L_{2}}=0$,

$\left(x /\left\{x_{2}\right\}, t\right) \in \Omega_{1 x /\left\{x_{2}\right\}} \times\left[0, t_{E N D}\right]$,

$\left.D_{13} \frac{\partial c_{1}(x, t)}{\partial x_{3}}\right|_{x_{3}=0^{+}}-\left.\lambda_{13} c_{1}(x, t)\right|_{x_{3}=0^{+}}=0$,

$\left(x /\left\{x_{3}\right\}, t\right) \in \Omega_{1 x /\left\{x_{3}\right\}} \times\left[0, t_{E N D}\right]$,

- as well as two conditions

$\left.c_{1}(x, t)\right|_{x_{3}=H_{1}^{-}}=\left.c_{2}(x, t)\right|_{x_{3}=H_{1}^{+}}$,

$\left(x_{1}, x_{2}, t\right) \in\left[0, L_{1}\right] \times\left[0, L_{2}\right] \times\left[0, t_{E N D}\right]$,

$\left.D_{13} \frac{\partial c_{1}(x, t)}{\partial x_{3}}\right|_{x_{3}=H_{1}^{-}}=\left.D_{23} \frac{\partial c_{2}(x, t)}{\partial x_{3}}\right|_{x_{3}=H_{1}^{+}}$,

$\left(x_{1}, x_{2}, t\right) \in\left[0, L_{1}\right] \times\left[0, L_{2}\right] \times\left[0, t_{E N D}\right]$,

where the function $c_{2}(x, t)$ is defined in the domain $\Omega_{2 x} \times\left[0, t_{E N D}\right]$ and is a nontrivial solution of the problem

AP2 stated below.

Auxiliary problem AP2. It is required to find the function $0 \not \equiv c_{2}(x, t): \Omega_{2 x} \times\left[0, t_{E N D}\right] \rightarrow \mathrm{i}^{1}$ that satisfies:

- homogeneous equation

$\frac{\partial c_{2}(x, t)}{\partial t}=\sum_{j=1}^{3} D_{2 j} \frac{\partial^{2} c_{2}(x, t)}{\partial x_{j}^{2}}$,

$(x, t) \in \operatorname{int} \Omega_{2 x} \times\left(0, t_{E N D}\right]$,

- heterogeneous initial condition

$\left.c_{2}(x, t)\right|_{t=0^{+}}=c_{20}(x), x \in \Omega_{2 x}$,

- homogeneous boundary conditions

$\left.\frac{\partial c_{2}(x, t)}{\partial x_{1}}\right|_{x_{1}=0^{+}}=0$,

$\left(x /\left\{x_{1}\right\}, t\right) \in \Omega_{2 x /\left\{x_{1}\right\}} \times\left[0, t_{E N D}\right]$,

$\left.D_{21} \frac{\partial c_{2}(x, t)}{\partial x_{1}}\right|_{x_{1}=L_{1}^{-}}+\left.\lambda_{21} c_{2}(x, t)\right|_{x_{1}=L_{1}^{-}}=0$,

$\left(x /\left\{x_{1}\right\}, t\right) \in \Omega_{2 x /\left\{x_{1}\right\}} \times\left[0, t_{E N D}\right]$,

$\left.\frac{\partial c_{2}(x, t)}{\partial x_{2}}\right|_{x_{2}=0^{+}}=0,\left(x /\left\{x_{2}\right\}, t\right) \in \Omega_{2 x /\left\{x_{2}\right\}} \times\left[0, t_{E N D}\right]$,

$\left.D_{22} \frac{\partial c_{2}(x, t)}{\partial x_{2}}\right|_{x_{2}=L_{2}}+\left.\lambda_{22} c_{1}(x, t)\right|_{x_{2}=L_{2}}=0$,

$\left(x /\left\{x_{2}\right\}, t\right) \in \Omega_{2 x /\left\{x_{2}\right\}} \times\left[0, t_{E N D}\right]$,
$\left.D_{23} \frac{\partial c_{2}(x, t)}{\partial x_{3}}\right|_{x_{3}=L_{3}^{-}}+\left.\lambda_{23} c_{2}(x, t)\right|_{x_{3}=L_{3}}=0$,

$\left(x /\left\{x_{3}\right\}, t\right) \in \Omega_{2 x /\left\{x_{3}\right\}} \times\left[0, t_{E N D}\right]$.

\section{B. Partial investigation of the first auxiliary} boundary-value problem

First of all, we note that the use of the phrase "partial research" in the titles of the current and next subsections is related to the interconnectedness of the auxiliary problems AP1 and AP2: as it will be seen in subsections B and $\mathrm{C}$ of this section, a full research of AP1 is impossible without research of AP2, and vice versa.

So, first consider the AP1 problem, a non-trivial solution of which will be sought in the form

$c_{1}(x, t)=X_{11}\left(x_{1}\right) X_{12}\left(x_{2}\right) X_{13}\left(x_{3}\right) T_{1}(t)$,

where the essence of the requirements $X_{1 j}\left(x_{j}\right) \not \equiv 0, j=\overline{1,3}$ and $T_{1}(t) \not \equiv 0$ is obvious.

Taking into account representation (25) in the equation (9), we get

$\frac{T_{1}^{\prime}(t)}{T_{1}(t)}=\sum_{j=1}^{3} D_{1 j} \frac{X_{1 j}^{\prime \prime}\left(x_{j}\right)}{X_{1 j}\left(x_{j}\right)}$.

Since the left side of equality (26) depends only on the time variable $t$, and the right side depends only on spatial variables $x=\left(x_{1}, x_{2}, x_{3}\right)$, equality (26) is possible only if both sides of it are equal to the same constant, which we will denote by $-\mu_{1}$, without making any assumptions regarding the sign of the constant $\mu_{1}$. So, instead of (26) we can write the following two equations:

$T_{1}^{\prime}(t)+\mu_{1} T_{1}(t)=0$,

$\sum_{j=1}^{3} D_{1 j} \frac{X_{1 j}^{\prime \prime}\left(x_{j}\right)}{X_{1 j}\left(x_{j}\right)}+\mu_{1}=0$

First we deal with equation (28), and then we return to equation (27). Alternately differentiating equation (28) with respect to variables $x_{1}, x_{2}$ and $x_{3}$, we obtain $\frac{d}{d x_{j}}\left(D_{1 j} \frac{X_{1 j}^{\prime \prime}\left(x_{j}\right)}{X_{1 j}\left(x_{j}\right)}\right)=0, \forall j=\overline{1,3}$, from which it follows that $D_{1 j} \frac{X_{1 j}^{\prime \prime}\left(x_{j}\right)}{X_{1 j}\left(x_{j}\right)}, \forall j=\overline{1,3}$ are constants: $D_{1 j} \frac{X_{1 j}^{\prime \prime}\left(x_{j}\right)}{X_{1 j}\left(x_{j}\right)}=\mu_{1 j}, \forall j=\overline{1,3}$, where $\mu_{1}=\sum_{j=1}^{3} \mu_{1 j}$, i.e. new constants $\mu_{11}, \mu_{12}, \mu_{13}$ are constituent constants of the 
initial constant $\mu_{1}$, appearing in equations (27) and (28). So, we obtained the following homogeneous equations of the same type:

$$
\begin{aligned}
& D_{11} X_{11}^{\prime \prime}\left(x_{1}\right)+\mu_{11} X_{11}\left(x_{1}\right)=0, \\
& D_{12} X_{12}^{\prime \prime}\left(x_{2}\right)+\mu_{12} X_{12}\left(x_{2}\right)=0, \\
& D_{13} X_{13}^{\prime \prime}\left(x_{3}\right)+\mu_{13} X_{13}\left(x_{3}\right)=0,
\end{aligned}
$$

which are related only by the fact that $\mu_{1}=\sum_{j=1}^{3} \mu_{1 j}$. As the constant's $\mu_{1}$ sign still is unknown to us, we also have no information about constants' $\mu_{11}, \mu_{12}, \mu_{13}$ signs.

Further, the substitution of (25) to the boundary conditions (11)(15) gives us the following boundary conditions:

- for the function $X_{11}\left(x_{1}\right)$ two boundary conditions: $\left\{\begin{array}{l}X_{11}^{\prime}(0)=0, \\ D_{11} X_{11}^{\prime}\left(L_{1}\right)+\lambda_{11} X_{11}\left(L_{1}\right)=0 ;\end{array}\right.$

- for the function $X_{12}\left(x_{2}\right)$ again two boundary conditions:

$\left\{\begin{array}{l}X_{12}^{\prime}(0)=0, \\ D_{12} X_{12}^{\prime}\left(L_{2}\right)+\lambda_{12} X_{12}\left(L_{2}\right)=0\end{array}\right.$

- for the function $X_{13}\left(x_{3}\right)$ one boundary condition:

$D_{13} X_{13}^{\prime}(0)-\lambda_{13} X_{13}(0)=0$.

Consequently, the combination of equation (29) and boundary conditions (32), the combination of equation (30) and boundary conditions (33), and finally the combination of equation (31) and boundary condition (34) generate the following three SturmLiouville problems [9], [10], the first two of which are complete problems (in the sense that they have a complete formulation: each of them has a second-order ordinary differential equation and two boundary conditions are given), and the third problem is an incomplete problem (one boundary condition is missing):

$$
\begin{aligned}
& \left\{\begin{array}{l}
D_{11} X_{11}^{\prime \prime}\left(x_{1}\right)+\mu_{11} X_{11}\left(x_{1}\right)=0, x_{1} \in\left(0, L_{1}\right), \\
X_{11}^{\prime}(0)=0 \\
D_{11} X_{11}^{\prime}\left(L_{1}\right)+\lambda_{11} X_{11}\left(L_{1}\right)=0
\end{array}\right. \\
& \left\{\begin{array}{l}
D_{12} X_{12}^{\prime \prime}\left(x_{2}\right)+\mu_{12} X_{12}\left(x_{2}\right)=0, x_{2} \in\left(0, L_{2}\right), \\
X_{12}^{\prime}(0)=0 \\
D_{12} X_{12}^{\prime}\left(L_{2}\right)+\lambda_{12} X_{12}\left(L_{2}\right)=0
\end{array}\right. \\
& \left\{\begin{array}{l}
D_{13} X_{13}^{\prime \prime}\left(x_{3}\right)+\mu_{13} X_{13}\left(x_{3}\right)=0, x_{3} \in\left(0, H_{1}\right), \\
D_{13} X_{13}^{\prime}(0)-\lambda_{13} X_{13}(0)=0
\end{array}\right.
\end{aligned}
$$

We will have to solve all three SturmLiouville problems in turn (35)(37): our goal is to find their non- trivial solutions $X_{1 j}\left(x_{j}\right) \not \equiv 0, j=\overline{1,3}$.

It is not difficult to show that in the case, when $\mu_{11} \leq 0$, the problem (35) has only a trivial solution. Therefore, in problem (35) only the case $\mu_{11}>0$ should be considered, and in this case the general solution of the problem (35) is the following function

$$
X_{11}\left(x_{1}\right)=A_{11} \cos \left(\sqrt{\frac{\mu_{11}}{D_{11}}} x_{1}\right), x_{1} \in\left[0, L_{1}\right]
$$

where $A_{11}$ is an arbitrary constant.

Function $X_{11}\left(x_{1}\right), x_{1} \in\left[0, L_{1}\right]$, defined by formula (38), is called eigenfunction of the SturmLiouville problem (for instance, see [9] as well as [6]), and it corresponds to the eigenvalue $\mu_{11}=D_{11}\left(\frac{\alpha_{1}}{L_{1}}\right)^{2}>0$, where $\alpha_{1}$ is a positive root of the transcendental equation

$\alpha_{1} \operatorname{tg}\left(\alpha_{1}\right)=\frac{\lambda_{11} L_{1}}{D_{11}}$

Since the transcendental equation (39) has an infinite number of solutions, we can say that the SturmLiouville problem (35) has an infinite number of eigenvalues

$\mu_{11 n}=D_{11}\left(\frac{\alpha_{1 n}}{L_{1}}\right)^{2}>0$,

to which the following eigenfunctions correspond

$X_{11 n}\left(x_{1}\right)=A_{11 n} \cos \left(\sqrt{\frac{\mu_{11 n}}{D_{11}}} x_{1}\right), x_{1} \in\left[0, L_{1}\right]$,

and each of them is determined with precision to an arbitrary constant $A_{11 n}$. In (41) number $\alpha_{1 n}$ is n-th $(n \in ¥)$ positive root of the transcendental equation (39), and, hereinafter, speaking of the ordinal numbers of the positive roots of the transcendental equation, we will mean their ordering in non-decreasing order: $\alpha_{11} \leq \alpha_{12} \leq \alpha_{13} \leq \mathrm{K}$

Because of the fact that the SturmLiouville problem (36) differs from the problem (35) only by the coefficients $D_{12}$ and $\lambda_{12}$, we can write, fully following the results of the study of the problem (35) obtained above, that the eigenfunctions of the SturmLiouville problem (36) are the functions

$X_{12 m}\left(x_{2}\right)=A_{12 m} \cos \left(\sqrt{\frac{\mu_{12 m}}{D_{12}}} x_{2}\right), x_{2} \in\left[0, L_{2}\right]$,

where $A_{12 m}$ are some constants; $\mu_{12 m}=D_{12}\left(\frac{\beta_{1 m}}{L_{2}}\right)^{2}>0$ are eigenvalues, $\beta_{1 m}$ is m-th 
$(m \in ¥)$ positive root of the transcendental equation

$\beta_{1} \operatorname{tg}\left(\beta_{1}\right)=\frac{\lambda_{12} L_{2}}{D_{12}}$.

Now we investigate the incomplete SturmLiouville problem (37). It is easy to check that when $\mu_{13}>0$ (in the case of $\mu_{13} \leq 0$ problem (37) has only trivial solution) incomplete problem (37) has general solution

$$
\begin{aligned}
& X_{13}\left(x_{3}\right)=A_{13}\left\{\sin \left(\sqrt{\frac{\mu_{13}}{D_{13}}} x_{3}\right)\right. \\
& \left.+\frac{\sqrt{\mu_{13} D_{13}}}{\lambda_{13}} \cos \left(\sqrt{\frac{\mu_{13}}{D_{13}}} x_{3}\right)\right\}, x_{3} \in\left[0, H_{1}\right],
\end{aligned}
$$

which is called the SturmLiouville incomplete problem eigenfunction (37) corresponding to the eigenvalue $\mu_{13}$ (not yet found); $A_{13}$ is an arbitrary constant. To find eigenvalue $\mu_{1,3}$, we should refer to conditions (16), (17), in which another function is involved - the desired function $c_{2}(x, t),(x, t) \in \Omega_{2 x} \times\left[0, t_{E N D}\right]$ of auxiliary problem AP2. In other words, to find the eigenvalues and the corresponding eigenfunctions of the incomplete SturmLiouville problem (37), we will need to investigate the auxiliary problem AP2, which we will do in subsection $\mathrm{C}$ of this section.

Recall that in the course of studying the AP1 problem (still unfinished), we found out that all the eigenvalues of problems (35)(37) of SturmLiouville are positive. Therefore, the constant $\mu_{1}$ from (27) and (28), which is the sum of these eigenvalues, is also positive:

$$
\begin{aligned}
& 0<\underset{\mu_{1} m m}{\mu_{\mu_{1}}}=\mu_{11 n}+\mu_{12 m}+\mu_{13} \\
& =D_{11}\left(\frac{\alpha_{1 n}}{L_{1}}\right)^{2}+D_{12}\left(\frac{\beta_{1 m}}{L_{2}}\right)^{2}+\mu_{13}, \forall n, m \in ¥,
\end{aligned}
$$

where $\alpha_{1 n}$ and $\beta_{1 m}$ are n-th $(n \in ¥)$ and m-th $(m \in ¥)$ positive roots of the transcendental equations (39) and (43), respectively; constituent constant $\mu_{13}>0$, which is the eigenvalue of the incomplete SturmLiouville problem (37), has not yet been found (it means that the eigenfunction $X_{13}\left(x_{3}\right), x_{3} \in\left[0, H_{1}\right]$, having a formal representation in the form (44) and corresponding to this eigenvalue, is not uniquely determined).

\section{Partial investigation of the second auxiliary boundary-value problem}

We will look for nontrivial solution of the AP2 problem in the following form

$c_{2}(x, t)=X_{21}\left(x_{1}\right) \cdot X_{22}\left(x_{2}\right) \cdot X_{23}\left(x_{3}\right) \cdot T_{2}(t)$,

where meaning of requirements $X_{2 j}\left(x_{j}\right) \not \equiv 0, j=\overline{1,3}$ and $T_{2}(t) \not \equiv$ is obvious. By analogy with the previous subsection B, given the representation (46) in equation
(18), we obtain

$$
\begin{aligned}
& T_{2}^{\prime}(t)+\mu_{2} T_{2}(t)=0, \\
& \sum_{j=1}^{3} D_{2 j} \cdot \frac{X_{2 j}^{\prime \prime}\left(x_{j}\right)}{X_{2 j}\left(x_{j}\right)}+\mu_{2}=0,
\end{aligned}
$$

where $\mu_{2}$ is the same constant $\mu_{1}$ as in equations (27), (28), i.e. $\mu_{2}=\mu_{1}$ (for convenience, we will use the notation $\mu_{2}$, knowing that $\mu_{2}=\mu_{1}$ ).

First we deal with equation (48), and then we return to equation (47). We already know that constants $\mu_{1}$ and $\mu_{2}$ in equations (28) and (48) coincide. However, we do not have the right to require that in three homogeneous equations of the same type

$D_{21} X_{21}^{\prime \prime}\left(x_{1}\right)+\mu_{21} X_{21}\left(x_{1}\right)=0$,
$D_{22} X_{22}^{\prime \prime}\left(x_{2}\right)+\mu_{22} X_{22}\left(x_{2}\right)=0$,
$D_{23} X_{23}^{\prime \prime}\left(x_{3}\right)+\mu_{23} X_{23}\left(x_{3}\right)=0$,

which directly follow from equation (48) (see the transition procedure from equation (28) to equations (29) (31)), constants $\mu_{21}, \mu_{22}$ and $\mu_{23}$, whose sum gives $\mu_{2}$ (i.e. $\left.\mu_{2}=\sum_{j=1}^{3} \mu_{2 j}\right)$, coincide with the previous constituent constants $\mu_{11}, \mu_{12}, \mu_{13}$ (values $\mu_{11}$ and $\mu_{12}$ are already determined, and value $\mu_{13}$ will be determined in this subsection). The reason for this circumstance (i.e. the fact that $\mu_{1}=\mu_{2}$, but $\mu_{1 j} \neq \mu_{2 j}, j=\overline{1,3}$ ) is due to the fact that the coefficients $D_{1 j}(j=\overline{1,3})$ in equations (29)(31) differ from the corresponding coefficients $D_{2 j}(j=\overline{1,3})$ in equations (49)(51). In other words, in the equations (49) (51) constants $\mu_{21}, \mu_{22}, \mu_{23}$, where $\sum_{j=1}^{3} \mu_{2 j}=\mu_{2}$, are still unknown constants and need to be determined. Finally, we note that equations of the same type (49)(51) are related only by the fact that $\mu_{2}=\sum_{j=1}^{3} \mu_{2 j}$. Further, the substitution of representations (46) to the boundary conditions (20)(24) gives us the following boundary conditions:

- for function $X_{21}\left(x_{1}\right)$ two boundary conditions: $\left\{\begin{array}{l}X_{21}^{\prime}(0)=0, \\ D_{21} X_{21}^{\prime}\left(L_{1}\right)+\lambda_{21} X_{21}\left(L_{1}\right)=0 ;\end{array}\right.$

- for function $X_{22}\left(x_{2}\right)$ again two boundary conditions:

$\left\{\begin{array}{l}X_{22}^{\prime}(0)=0, \\ D_{22} X_{22}^{\prime}\left(L_{2}\right)+\lambda_{22} X_{22}\left(L_{2}\right)=0\end{array}\right.$ 
- for function $X_{23}\left(x_{3}\right)$ one boundary condition: $D_{23} X_{23}^{\prime}\left(L_{3}\right)+\lambda_{23} X_{23}\left(L_{3}\right)=0$.

Consequently, the appropriate combination of equations (49)(51) and boundary conditions (52)(54) again give us the following three SturmLiouville problems, the first two of which are complete problems, and the third problem, just like problem (37) is not a complete problem:

$$
\begin{aligned}
& \left\{\begin{array}{l}
D_{21} X_{21}^{\prime \prime}\left(x_{1}\right)+\mu_{21} X_{21}\left(x_{1}\right)=0, x_{1} \in\left(0, L_{1}\right), \\
X_{21}^{\prime}(0)=0, \\
D_{21} X_{21}^{\prime}\left(L_{1}\right)+\lambda_{21} X_{21}\left(L_{1}\right)=0 ;
\end{array}\right. \\
& \left\{\begin{array}{l}
D_{22} X_{22}^{\prime \prime}\left(x_{2}\right)+\mu_{22} X_{22}\left(x_{2}\right)=0, x_{2} \in\left(0, L_{2}\right), \\
X_{22}^{\prime}(0)=0 \\
D_{22} X_{22}^{\prime}\left(L_{2}\right)+\lambda_{22} X_{22}\left(L_{2}\right)=0 ;
\end{array}\right. \\
& \left\{\begin{array}{l}
D_{23} X_{23}^{\prime \prime}\left(x_{3}\right)+\mu_{23} X_{23}\left(x_{3}\right)=0, x_{3} \in\left(H_{1}, L_{3}\right), \\
D_{23} X_{23}^{\prime}\left(L_{3}\right)+\lambda_{23} X_{23}\left(L_{3}\right)=0 .
\end{array}\right.
\end{aligned}
$$

Almost completely following the reasoning from subsection B in the study of problems (35) and (36), with respect to complete problems (55) and (56) of SturmLiouville, we can assert the following statements without detailed derivation:

- The complete problem (55) of SturmLiouville has eigenvalues

$\mu_{21 k}=D_{21}\left(\frac{\alpha_{2 k}}{L_{1}}\right)^{2}>0, k \in ¥$,

to which the following eigenfunctions correspond

$$
X_{21 k}\left(x_{1}\right)=A_{21 k} \cos \left(\sqrt{\frac{\mu_{21 k}}{D_{21}}} x_{1}\right), x_{1} \in\left[0, L_{1}\right],
$$

and each of them is determined with precision to an arbitrary constant $A_{21 k}$. In (59) number $\alpha_{2 k}$ is k-th $(k \in ¥)$ positive root of the transcendental equation $\alpha_{2} \operatorname{tg}\left(\alpha_{2}\right)=\frac{\lambda_{21} L_{1}}{D_{21}}$.

- The complete problem (56) of SturmLiouville has eigenvalues

$$
\mu_{22 p}=D_{22}\left(\frac{\beta_{2 p}}{L_{2}}\right)^{2}>0, p \in ¥,
$$

to which the following eigenfunctions correspond

$$
X_{22 p}\left(x_{2}\right)=A_{22 p} \cos \left(\sqrt{\frac{\mu_{22 p}}{D_{22}}} x_{2}\right), \forall x_{2} \in\left[0, L_{2}\right] \text {, }
$$

and each of them is determined with precision to an arbitrary constant $A_{22 p}$. In (62) number $\beta_{2 p}$ is $\mathrm{p}$-th $(p \in ¥)$ positive root of the transcendental equation

$\beta_{2} \operatorname{tg}\left(\beta_{2}\right)=\frac{\lambda_{22} L_{2}}{D_{22}}$.

Now let us study the incomplete SturmLiouville problem (57). It is easy to check that when $\mu_{23}>0$ (in the case of $\mu_{23} \leq 0$ problem (57) has only trivial solution) the general solution of (57) is function

$$
\begin{aligned}
& X_{23}\left(x_{3}\right)=A_{23}\left\{\sin \left(\sqrt{\frac{\mu_{23}}{D_{23}}} x_{3}\right)\right. \\
& \left.-\cos \left(\sqrt{\frac{\mu_{23}}{D_{23}}} x_{3}\right) \operatorname{tg}\left(\sqrt{\frac{\mu_{23}}{D_{23}}} L_{3}+\theta\right)\right\}, x_{3} \in\left[H_{1}, L_{3}\right],
\end{aligned}
$$

where $A_{23}$ is an arbitrary constant; $\theta=\operatorname{arctg}\left(\frac{\sqrt{\mu_{23} D_{23}}}{\lambda_{23}}\right)$.

Function $X_{23}\left(x_{3}\right)$, defined by formula (64), is called the eigenfunction of the incomplete SturmLiouville problem (57) corresponding to the eigenvalue $\mu_{23}$. Recall that in the course of studying the AP2 problem (still unfinished), we found out that all the eigenvalues of problems (55)(57) of SturmLiouville are positive. Therefore, the constant $\mu_{2}$ from (47) and (48), which is the sum of these eigenvalues, is also positive:

$$
\begin{aligned}
& 0<\underbrace{\mu_{2} k}_{\mu_{2}}=\mu_{21 k}+\mu_{22 p}+\mu_{23} \\
& =D_{21}\left(\frac{\alpha_{2 k}}{L_{1}}\right)^{2}+D_{22}\left(\frac{\beta_{2 p}}{L_{2}}\right)^{2}+\mu_{23}, \forall k, p \in ¥,
\end{aligned}
$$

where $\alpha_{2 k}$ and $\beta_{2 p}$ are k-th $(k \in ¥)$ and $\mathrm{p}$-th $(p \in ¥)$ positive roots of the transcendental equations (60) and (63), respectively; constituent constant $\mu_{23}>0$, which is eigenvalue of incomplete SturmLiouville problem (57), is still unknown (it means that the eigenfunction $X_{23}\left(x_{3}\right), x_{3} \in\left[H_{1}, L_{3}\right]$, having a formal representation in the form (64) and corresponding to this eigenvalue, is not uniquely determined).

So, within the framework of the study of auxiliary problems AP1 and AP2, by this time the eigenvalues $\mu_{13}$ and $\mu_{23}$, remain uncertain and, therefore, same thing can be said about their corresponding eigenfunctions $X_{13}\left(x_{3}\right), x_{3} \in\left[0, H_{1}\right]$ and $X_{23}\left(x_{3}\right), x_{3} \in\left[H_{1}, L_{3}\right] ; \quad$ in addition, it is necessary to clarify the choice of constants $A_{11 n}(n \in ¥), \quad A_{12 m}(m \in ¥), \quad A_{13}, \quad A_{21 k}(k \in ¥)$, $A_{22 p}(p \in ¥), A_{23}$; finally, it is required to find functions $T_{1}(t)$ and $T_{2}(t)$, which satisfy equations (27) and (47).

\section{Using the matching conditions, and the complete solving the both auxiliary problems}

Recall that in the course of studying the auxiliary problems AP1 and AP2, we did not use matching conditions (16) and (17), and now it is time to use these conditions to find eigenvalues $\mu_{13}$ and $\mu_{23}$, and redefine the corresponding eigenfunctions $X_{13}\left(x_{3}\right), x_{3} \in\left[0, H_{1}\right]$ and $X_{23}\left(x_{3}\right), x_{3} \in\left[H_{1}, L_{3}\right]$, formally represented by formulas (44) and (64)), respectively. For this purpose, we first note that if equality 


$$
\underbrace{T_{1}(t) \prod_{j=1}^{3} X_{1 j}\left(x_{j}\right)}_{c_{1}(x, t)}=\underbrace{T_{1}(t) \prod_{j=1}^{3} X_{2 j}\left(x_{j}\right)}_{c_{2}(x, t)}
$$

holds for $\forall x_{j} \in\left[0, L_{j}\right], j=1,2, \forall t \in\left[0, T_{E N D}\right]$ and $\forall x_{3} \in\left(H_{1}-\varepsilon, H_{1}+\varepsilon\right), 0<\varepsilon=1$, then it means that $X_{13}\left(x_{3}\right)=C X_{23}\left(x_{3}\right), 0<\forall \varepsilon=1$, where $C \neq 0$ is an arbitrary constant, which for convenience we choose as $\frac{A_{13}}{A_{23}}$, i.e. $C=\frac{A_{13}}{A_{23}}$ (such a choice of a constant is legitimate because of its arbitrariness, and, moreover, nothing will change from such (or other) choice). Now, having this fact, substituting representations (25) and (46) to matching conditions (16) and (17), we obtain

$$
\begin{gathered}
\left.A_{23} X_{13}\left(x_{3}\right)\right|_{x_{3}=H_{1}^{-}}=A_{13} X_{23}\left(x_{3}\right)_{x_{3}=H_{1}^{+}}, \\
\left.A_{23} D_{13} X_{13}^{\prime}\left(x_{3}\right)\right|_{x_{3}=H_{1}^{-}}=\left.A_{13} D_{23} X_{13}^{\prime}\left(x_{3}\right)\right|_{x_{3}=H_{1}^{+}} .
\end{gathered}
$$

Taking into account formulas (44) and (64) in these two equalities, after performing the necessary calculations and transformations, gives us the following results: the desired eigenvalue $\mu_{23}$ from the SturmLiouville problem (57) are found by the formula

$$
\mu_{23}=D_{23}\left(\frac{\gamma}{H_{2}}\right)^{2}>0 \text {, }
$$

and then the desired eigenvalue $\mu_{13}$ from the SturmLiouville problem (37) is calculated by the formula $\mu_{13}=\mu_{21}+\mu_{22}+\mu_{23}-\mu_{11}-\mu_{12}$,

whose right side contains already found eigenvalues of SturmLiouville problems (35), (36), (55), (56).

In the formula (66) parameter $\gamma$ is positive root of the transcendental equation

$$
\begin{gathered}
\frac{\sqrt{D_{23} \gamma^{2}+\xi}}{\gamma} \operatorname{tg}\left(\gamma+\operatorname{arctg}\left(\frac{D_{23} \gamma}{H_{2} \lambda_{23}}\right)\right)=g(\gamma), \\
g(\gamma)=\frac{D_{23}}{\sqrt{D_{13}}} \frac{\sqrt{D_{13}\left(D_{23} \gamma^{2}+\xi\right)}+H_{2} \lambda_{13} \operatorname{tg}\left(\frac{H_{1}}{H_{2}} \sqrt{\frac{D_{23} \gamma^{2}+\xi}{D_{13}}}\right)}{\sqrt{D_{13}\left(D_{23} \gamma^{2}+\xi\right)} \operatorname{tg}\left(\frac{H_{1}}{H_{2}} \sqrt{\frac{D_{23} \gamma^{2}+\xi}{D_{13}}}\right)-H_{2} \lambda_{13}}, \\
\xi=H_{2}^{2}\left(\mu_{21}+\mu_{22}-\mu_{11}-\mu_{12}\right) .
\end{gathered}
$$

Since the transcendental equation (68) has an infinite number of solutions, we arrive at the following results:

- The SturmLiouville problem (57) has an infinite number of eigenvalues

$$
\begin{aligned}
& \mu_{23 q}=D_{23}\left(\frac{\gamma_{q}}{H_{2}}\right)^{2}, q \in ¥, \\
& q=q(n, m, k, p), \forall n, m, k, p \in ¥,
\end{aligned}
$$

to which the following eigenfunctions correspond

$$
\begin{aligned}
& X_{23 q}\left(x_{3}\right)=\sin \left(\sqrt{\frac{\mu_{23 q}}{D_{23}}} x_{3}\right) \\
& -\operatorname{tg}\left(\sqrt{\frac{\mu_{23 q}}{D_{23}}} L_{3}+\operatorname{arctg}\left(\frac{\sqrt{\mu_{23 q} D_{23}}}{\lambda_{23}}\right)\right) \times \\
& \times \cos \left(\sqrt{\frac{\mu_{23 q}}{D_{23}}} x_{3}\right), x_{3} \in\left[H_{1}, L_{3}\right] ;
\end{aligned}
$$

in (69) number $\gamma_{q}=\gamma_{q(n, m, k, p)}$ is q-th $(q \in ¥)$ positive root of the transcendental equation (68);

- The SturmLiouville problem (37) has an infinite number of eigenvalues

$$
\begin{aligned}
& \mu_{13 n m k p q}=\mu_{21 k}+\mu_{22 p}+\mu_{23 q}-\mu_{11 n}-\mu_{12 m}, \\
& q=q(n, m, k, p), \forall n, m, k, p \in ¥,
\end{aligned}
$$

to which the following eigenfunctions correspond

$$
\begin{aligned}
& X_{13 n m k p q}\left(x_{3}\right)=\frac{\sqrt{\mu_{13 n m k p q} D_{13}}}{\lambda_{13}} \cos \left(\sqrt{\frac{\mu_{13 n m k p q}}{D_{13}} x_{3}}\right) \\
& +\sin \left(\sqrt{\frac{\mu_{13 n m k p q}}{D_{13}}} x_{3}\right), x_{3} \in\left[0, H_{1}\right] .
\end{aligned}
$$

So, within the framework of the study of auxiliary problems AP1 and AP2, two sub-problems remain unfinished: the problem of finding functions $T_{1}(t)$ and $T_{2}(t)$, for whose solution one, first of all, needs to clarify/redefine formulas (45) and (65) for constants $\mu_{1}$ and $\mu_{2}$; problem of choosing constants $A_{11 n}(n \in ¥)$, $A_{12 m}(m \in ¥), \quad A_{21 k}(k \in ¥)$ and $A_{22 p}(p \in ¥)$, which appear in formulas (41), (42), (59) and (62), respectively. We start by clarifying/redefining formulas (45) and (65) for constants $\mu_{1}$ and $\mu_{2}$. Since constants $\mu_{1}$ and $\mu_{2}$ coincide, then it is sufficed to clarify the formula (45) for the constant $\mu_{1}$ only:

$$
\begin{aligned}
& 0<\underbrace{\mu_{1 n m k p q}}_{\mu_{1}=\mu_{2} \equiv \mu}=D_{21}\left(\frac{\alpha_{2 k}}{L_{1}}\right)^{2}+D_{22}\left(\frac{\beta_{2 p}}{L_{2}}\right)^{2} \\
& +D_{23}\left(\frac{\gamma_{q}}{H_{2}}\right)^{2}-D_{11}\left(\frac{\alpha_{1 n}}{L_{1}}\right)^{2}-D_{12}\left(\frac{\beta_{1 m}}{L_{2}}\right)^{2}
\end{aligned}
$$

Now we clarify the problem of choosing constants $A_{11 n}(n \in ¥), \quad A_{12 m}(m \in ¥), \quad A_{21 k}(k \in ¥) \quad$ and $A_{22 p}(p \in ¥)$, which appear in formulas (41), (42), (59) and (62), respectively. For this we use the fact that the system of eigenfunctions of the SturmLiouville problem forms an orthogonal system with some weight on the segment. Therefore, we can state that the system of functions $\left\{X_{11 n}\left(x_{1}\right)\right\}_{n \in ¥},\left\{X_{12 m}\left(x_{2}\right)\right\}_{m \in \sharp},\left\{X_{21 k}\left(x_{1}\right)\right\}_{k \in ¥}$, $\left\{X_{22 p}\left(x_{2}\right)\right\}_{p \in ¥}$, represented by formulas (41), (42), (59), (62), respectively, are orthogonal systems on segments $\left[0, L_{1}\right],\left[0, L_{2}\right],\left[0, L_{1}\right],\left[0, L_{2}\right]$, respectively. One of the 
reasonable constraints on choice of constants $A_{11 n}(n \in ¥)$, $A_{12 m}(m \in ¥), A_{21 k}(k \in ¥), A_{22 p}(p \in ¥)$, which appear in formulas (41), (42), (59), (62), respectively, is the requirement of orthonormality of systems $\left\{X_{11 n}\left(x_{1}\right)\right\}_{n \in \dddot{*}}$, $\left\{X_{12 m}\left(x_{2}\right)\right\}_{m \in \sharp},\left\{X_{21 k}\left(x_{1}\right)\right\}_{k \in \sharp},\left\{X_{22 p}\left(x_{2}\right)\right\}_{p \in ¥}$ of eigenfunctions. Another option (very simple, but less reasonable) of choosing constants is simply equating them to some number, for example, to 1 . In this paper, we choose the first option:

$$
\begin{aligned}
& X_{11 n}\left(x_{1}\right)=\sqrt{\frac{\lambda_{11}}{D_{11}}+\frac{\mu_{11 n}}{\lambda_{11}}} \sin \left(\sqrt{\frac{\mu_{11 n}}{D_{11}}} x_{1}\right), \\
& \forall x_{1} \in\left[0, L_{1}\right], \forall n \in ¥ . \\
& X_{12 m}\left(x_{2}\right)=\sqrt{\frac{\lambda_{12}}{D_{12}}+\frac{\mu_{12 m}}{\lambda_{12}}} \sin \left(\sqrt{\frac{\mu_{12 m}}{D_{12}}} x_{2}\right), \\
& \forall x_{2} \in\left[0, L_{2}\right], \forall m \in ¥ . \\
& X_{21 k}\left(x_{1}\right)=\sqrt{\frac{\lambda_{21}}{D_{21}}+\frac{\mu_{21 k}}{\lambda_{21}}} \sin \left(\sqrt{\frac{\mu_{21 k}}{D_{21}}} x_{1}\right), \\
& \forall x_{1} \in\left[0, L_{1}\right], \forall k \in ¥ . \\
& X_{22 p}\left(x_{1}\right)=\sqrt{\frac{\lambda_{22}}{D_{22}}+\frac{\mu_{22 p}}{\lambda_{22}}} \sin \left(\sqrt{\frac{\mu_{22 p}}{D_{22}}} x_{2}\right), \\
& \forall x_{2} \in\left[0, L_{2}\right], \forall p \in ¥ .
\end{aligned}
$$

Now we can proceed to solving the last problem in the framework of the study of auxiliary problems AP1 and AP2 - the problem of finding functions $T_{1}(t)$ and $T_{2}(t)$ from equations (27) and (47), respectively. If we consider equations (27) and (47) only from the position of time $t \in\left[0, t_{E N D}\right]$, rather than from the position of spatial variables, the solution of these equations is the function $T(t)=B e^{-\mu \cdot t}$, where $B$ is some coefficient that is not yet defined. Since number $\mu\left(=\mu_{1}=\mu_{2}\right)$ is determined by finally found formula (72), we can write

$$
T_{n m k p q}(t)=B_{n m k p q} e^{-\mu_{n m k p q} \cdot t}, \forall n, m, k, p, q \in ¥,
$$

where coefficients $B_{n m k p q}$ are to be determined taking into account spatial variables.

To satisfy conditions (10) and (19), in the formula (77) for the layer $\Omega_{1 x}$ there should be its own distinctive coefficients $B_{1 n m k p q}$, and for the layer $\Omega_{2 x}$ there should be its own distinctive constants $B_{2 n m k p q}$. Taking into account formula (73) for $X_{11 n}\left(x_{1}\right)$, formula (74) for $X_{12 m}\left(x_{2}\right)$, formula (71) for $X_{13 m m k q}\left(x_{3}\right)$, formula (75) for $X_{21 k}\left(x_{1}\right)$, formula (76) for $X_{22 p}\left(x_{2}\right)$, formula (70) for $X_{23 \text { mmkpq }}\left(x_{3}\right)$, formula (77) for $T_{\text {nmkpq }}(t)$ in representations (25) and (46), we obtain the following formula for the desired functions $c_{1}(x, t)$, where $(x, t) \in \Omega_{1 x} \times\left[0, t_{E N D}\right]$, and $c_{2}(x, t)$, where $(x, t) \in \Omega_{2 x} \times\left[0, t_{E N D}\right]:$

$c_{1}(x, t)=\sum_{n, m, k, p, q=1}^{+\infty} B_{1 n m k p q} e^{-\mu_{n m k p q} \cdot t} X_{1 n m k p q}(x)$,

where $\quad X_{1 n m k p q}(x)=X_{11 n}\left(x_{1}\right) X_{12 m}\left(x_{2}\right) X_{13 n m k p q}\left(x_{3}\right)$, $x_{1} \in\left[0, L_{1}\right], \quad x_{2} \in\left[0, L_{2}\right], \quad x_{3} \in\left[0, H_{1}\right], t \in\left[0, t_{E N D}\right]$, system of functions $\left\{X_{1 n m k p q}(x)\right\}_{n, m, k, p, q \in ¥}$ is an orthogonal system; $c_{2}(x, t)=\sum_{n, m, k, p, q=1}^{+\infty} B_{2 n m k p q} e^{-\mu_{m m p p q} \cdot t} X_{2 n m l p q}(x)$,

where $\quad X_{2 n m k p q}(x)=X_{21 k}\left(x_{1}\right) X_{22 p}\left(x_{2}\right) X_{23 n m k p q}\left(x_{3}\right)$, $x_{1} \in\left[0, L_{1}\right], \quad x_{2} \in\left[0, L_{2}\right], \quad x_{3} \in\left[H_{1}, L_{3}\right], \quad t \in\left[0, t_{E N D}\right]$, system of functions $\left\{X_{2 n m k p q}(x)\right\}_{n, m, k, p, q \in ¥}$ is an orthogonal system.

Obviously, the function $c_{1}(x, t)$, determined by the formula (78), satisfies all homogeneous boundary conditions (11)(15) of the auxiliary problem AP1, since they are satisfied by all members of the quadruple series in the right-hand side (78); similarly function $c_{2}(x, t)$, determined by the formula (79), satisfies all homogeneous boundary conditions (20)(24) of the auxiliary problem AP1, since they are satisfied by all members of the quadruple series in the right-hand side (79); in addition, these functions satisfy the matching conditions (16), (17), since functions $X_{13 n m k p q}\left(x_{3}\right)$ and $X_{23 n m k p q}\left(x_{3}\right)$, which are contained in each member of the quadruple series of (78) and (79), respectively, automatically satisfy the matching conditions (16), (17) - functions $X_{13 n m k p q}\left(x_{3}\right)$ and $X_{23 m m k p q}\left(x_{3}\right)$ were determined owing to the conditions (16), (17). Therefore, it remains to enforce functions $c_{1}(x, t)$ and $c_{2}(x, t)$ to satisfy the initial conditions (10) and (19), respectively.

The sought-for function $c_{1}(x, t)$, which is determined by formula (78), to satisfy initial condition (10), we obtain: $c_{10}(x)=\sum_{n, m, k, p, q=1}^{+\infty} B_{1 n m k p q} X_{1 n m k p q}(x)$.

Analogously, the sought-for function $c_{2}(x, t)$ determined by formula (79) to satisfy the initial condition (19), we obtain:

$$
c_{20}(x)=\sum_{n, m, k, p, q=1}^{+\infty} B_{2 n m k p q} X_{2 n m k p q}(x) .
$$

Let us by turns apply to (80) and (81) one of the fundamental theorems of mathematical physics -Steklov's Theorem on decomposability of any twice continuously differentiable function into absolutely and uniformly convergent series by orthogonal system of eigenfunctions of the SturmLiouville problem (first strictly proved in 
[11]; see also [10]): for $\forall n, m, k, p, q \in ¥$ there take place

$B_{1 n m k p q}=\frac{\int_{\Omega_{1 x}} c_{10}(y) X_{1 n m k p q}(y) d y}{\int_{\Omega_{1 z}} X_{1 n m k p q}^{2}(z) d z}$

$=\frac{\int_{0}^{L_{1}} d y_{1} \int_{0}^{L_{2}} d y_{2} \int_{0}^{H_{1}} c_{10}(y) X_{1 \text { nmkpq }}(y) d y_{3}}{\int_{0}^{L_{1}} d z_{1} \int_{0}^{L_{2}} d z_{2} \int_{0}^{H_{1}} X_{1 n m k p q}^{2}(z) d z_{3}}$,

$B_{2 n m k p q}=\frac{\int_{\Omega_{2 y}} c_{20}(y) X_{2 n m k p q}(y) d y}{\int_{\Omega_{2 z}} X_{2 n m k p q}^{2}(z) d z}$

$=\frac{\int_{0}^{L_{1}} d y_{1} \int_{0}^{L_{2}} d y_{2} \int_{H_{1}}^{L_{3}} c_{20}(y) X_{2 n m k p q}(y) d y_{3}}{\int_{0}^{L_{1}} d z_{1} \int_{0}^{L_{2}} d z_{2} \int_{H_{1}}^{L_{3}} X_{2 n m k p q}^{2}(z) d z_{3}}$.

It is not difficult to prove [6] that the function $c_{1}(x, t)$ defined by formulas (78), (82) is a continuously differentiable function by a variable $t$ in the interval $\left[0, t_{E N D}\right]$ and twice continuously differentiable function by variable $x$ for the layer $\Omega_{1 x}$, which satisfies the equation (9). Similarly, a function $c_{2}(x, t)$, defined by formulas (79), (83) is a continuously differentiable function for a variable in a segment and a twice continuously differentiable function (twice differentiable function) for a variable $t$ for the layer $\Omega_{2 x}$, which satisfies equation (18). Thus, the functions $c_{1}(x, t)$ and $c_{2}(x, t)$ are continuous functions for $\Omega_{1 x} \times\left[0, t_{E N D}\right]$ and $\Omega_{2 x} \times\left[0, t_{E N D}\right]$, respectively, and since these functions satisfy the matching conditions (16), (17), they are considered to be solutions of auxiliary problems AP1 and AP2, respectively. Thus, the study of auxiliary problems AP1 and AP2 is entirely completed, and now we can proceed to finding a solution for the original problem (1)-(8).

\section{E. Solving the original problem (1)(8)}

Obviously, substituting (82) in (78) and (83)

in (79), we get the following representations for the functions $c_{1}(x, t), \forall(x, t) \in \Omega_{1 x} \times\left[0, t_{E N D}\right]$ and $c_{2}(x, t), \forall(x, t) \in \Omega_{2 x} \times\left[0, t_{E N D}\right]:$

$c_{1}(x, t)=\int_{\Omega_{1 y}} G_{1}(x, y, t) c_{10}(y) d y$,

$G_{1}(x, y, t)=\sum_{n, m, k, p, q=1}^{+\infty} e^{-\mu_{n n k p q q} t} \frac{X_{1 n m k p q}(x) X_{1 n m l p q}(y)}{\int_{\Omega_{1 z}} X_{1 n m k p q}^{2}(z) d z}$,

$c_{2}(x, t)=\int_{\Omega_{2 y}} G_{2}(x, y, t) c_{20}(y) d y$,

$$
G_{2}(x, y, t)=\sum_{n, m, k, p, q=1}^{+\infty} e^{-\mu_{m m k p q} \cdot t} \frac{X_{2 n m k p q}(x) X_{2 n m k p q}(y)}{\int_{\Omega_{2 z}} X_{2 n m k p q}^{2}(z) d z},
$$

which are a more compact form for auxiliary problems AP1 and AP2 solutions, respectively. Each of the above introduced functions $G_{j}(x, y, t), j=1,2$ is a well-known and deeply studied Green's function [6], [10], [13], [14]. Our goal in this subsection is the analytical construction of the solution to the original problem (1)(8), using the Green's functions $G_{j}(x, y, t), j=1,2$. As you will be able to see below, after completing the study of auxiliary problems AP1, AP2, there is no difficulty in finding an analytical solution to the original problem (1)-(8): a more or less difficult part of the research for the problem considered in this paper is the study of auxiliary problems AP1 and AP2.

Let us formulate a new auxiliary problem, naming it NAP1: it is required to find solutions to the inhomogeneous equation (which coincides with equation (1) for $i=1$ )

$$
\begin{aligned}
& \frac{\partial c_{1}(x, t)}{\partial t}=\sum_{j=1}^{3} D_{1 j} \frac{\partial^{2} c_{1}(x, t)}{\partial x_{j}^{2}}+f_{1}(x, t), \\
& (x, t) \in \operatorname{int} \Omega_{1 x} \times\left(0, t_{E N D}\right],
\end{aligned}
$$

which satisfies the zero-initial condition

$$
\left.c_{1}(x, t)\right|_{t=0^{+}}=0, x \in \Omega_{1 x}
$$

and zero-boundary conditions (11)-(15) of the auxiliary problem AP1.

The desired solution of the NAP1 is the function

$c_{1}(x, t)=\int_{0}^{t} d \tau \int_{\Omega_{1 y}} G_{1}(x, y, t-\tau) f_{1}(y, \tau) d y$,

where the function $G_{1}(x, y, t-\tau)$ is the same Green function (85), in which instead of the argument $t$ there is an argument $t-\tau$.

Now let us formulate a new auxiliary problem NAP2: it is required to find solutions to the inhomogeneous equation (which coincides with equation (1) at $i=2$ )

$$
\begin{aligned}
& \frac{\partial c_{2}(x, t)}{\partial t}=\sum_{j=1}^{3} D_{2 j} \frac{\partial^{2} c_{2}(x, t)}{\partial x_{j}^{2}}+f_{2}(x, t), \\
& (x, t) \in \operatorname{int} \Omega_{2 x} \times\left(0, t_{E N D}\right],
\end{aligned}
$$

which satisfies the zero-initial condition

$$
\left.c_{2}(x, t)\right|_{t=0^{+}}=0, x \in \Omega_{2 x}
$$

and to zero-boundary conditions (20)-(24) of the auxiliary problem AP2.

The desired solution of the NAP2 is the function

$$
c_{2}(x, t)=\int_{0}^{t} d \tau \int_{\Omega_{2 y}} G_{2}(x, y, t-\tau) f_{2}(y, \tau) d y,
$$

where the function $G_{2}(x, y, t-\tau)$ is the same Green's function (87), in which instead of the argument $t$ there is an argument $t-\tau$.

Thus, the following functions that contain the 
corresponding right-hand sides of (84), (88) and (86), (89), give us a solution to problem (1)-(8) provided that $c_{j 1}\left(x_{2}, x_{3}, t\right) \equiv 0, \quad a_{j 1}\left(x_{2}, x_{3}, t\right) \equiv 0, \quad c_{j 2}\left(x_{1}, x_{3}, t\right) \equiv 0$, $a_{j 2}\left(x_{1}, x_{3}, t\right) \equiv 0, a_{j 3}\left(x_{1}, x_{2}, t\right) \equiv 0$ for $\forall j=1,2$ :

$c_{j}(x, t)=\int_{\Omega_{j y}} G_{j}(x, y, t) c_{j 0}(y) d y$

$$
+\int_{0}^{t} d \tau \int_{\Omega_{j y}} G_{j}(x, y, t-\tau) f_{j}(y, \tau) d y, j=1,2
$$

Therefore, to complete our study, it remains to find a solution to problem (1)-(8), provided that $f_{j}(x, y, t) \equiv 0$ and $c_{j 0}(x) \equiv 0$ for $\forall j=1,2$, and then, add the solution found to the right side of the formula (90). To achieve this, it is necessary to use the multiplicative property of the Green functions as well as the properties of the Dirac delta function [14], [15]:

- function $c_{1}(x, t)$, which is defined as

$c_{1}(x, t)=\left.\int_{0}^{t} d \tau \int_{0}^{L_{2}} d y_{2} \int_{0}^{H_{1}} G_{1}(x, y, t-\tau)\right|_{y_{1}=0} \frac{-c_{11}\left(y_{2}, y_{3}, \tau\right)}{D_{11}} d y_{3}$

$+\left.\int_{0}^{t} d \tau \int_{0}^{L_{2}} d y_{2} \int_{0}^{H_{1}} G_{1}(x, y, t-\tau)\right|_{y_{1}=L_{1}} a_{11}\left(y_{2}, y_{3}, \tau\right) d y_{3}$

$+\left.\int_{0}^{t} d \tau \int_{0}^{L_{1}} d y_{1} \int_{0}^{H_{1}} G_{1}(x, y, t-\tau)\right|_{y_{2}=0} \frac{-a_{11}\left(y_{1}, y_{3}, \tau\right)}{D_{12}} d y_{3}$

$+\left.\int_{0}^{t} d \tau \int_{0}^{L_{1}} d y_{1} \int_{0}^{H_{1}} G_{1}(x, y, t-\tau)\right|_{y_{2}=L_{2}} a_{12}\left(y_{1}, y_{3}, \tau\right) d y_{3}$

$+\left.\int_{0}^{t} d \tau \int_{0}^{L_{1}} d y_{1} \int_{0}^{L_{2}} G_{1}(x, y, t-\tau)\right|_{y_{3}=0} \frac{-a_{13}\left(y_{1}, y_{2}, \tau\right)}{D_{13}} d y_{2}$,

is a solution to problem (1)-(8) at $i=1$ under conditions $f_{1}(x, t) \equiv 0, c_{10}(x) \equiv 0$ (i.e. with homogeneous equation (1), zero initial condition (2), and non-zero boundary conditions (3)-(7));

- function $c_{2}(x, t)$, which is defined as

$c_{2}(x, t)=\left.\int_{0}^{t} d \tau \int_{0}^{L_{2}} d y_{2} \int_{H_{1}}^{L_{3}} G_{2}(x, y, t-\tau)\right|_{y_{1}=0} \frac{-c_{21}\left(y_{2}, y_{3}, \tau\right)}{D_{21}} d y_{3}$

$+\left.\int_{0}^{t} d \tau \int_{0}^{L_{2}} d y_{2} \int_{H_{1}}^{L_{3}} G_{2}(x, y, t-\tau)\right|_{y_{1}=L_{1}} a_{21}\left(y_{2}, y_{3}, \tau\right) d y_{3}$

$+\left.\int_{0}^{t} d \tau \int_{0}^{L_{1}} d y_{1} \int_{H_{1}}^{L_{3}} G_{2}(x, y, t-\tau)\right|_{y_{2}=0} \frac{-a_{11}\left(y_{1}, y_{3}, \tau\right)}{D_{22}} d y_{3}$

$+\left.\int_{0}^{t} d \tau \int_{0}^{L_{1}} d y_{1} \int_{H_{1}}^{L_{3}} G_{2}(x, y, t-\tau)\right|_{y_{2}=L_{2}} a_{22}\left(y_{1}, y_{3}, \tau\right) d y_{3}$

$+\left.\int_{0}^{t} d \tau \int_{0}^{L_{1}} d y_{1} \int_{0}^{L_{2}} G_{2}(x, y, t-\tau)\right|_{y_{3}=L_{3}} \frac{-a_{23}\left(y_{1}, y_{2}, \tau\right)}{D_{23}} d y_{2}$,

is a solution to problem (1)-(8) at $i=2$ under conditions $f_{2}(x, t) \equiv 0, \quad c_{20}(x) \equiv 0$ (i.e. with homogeneous equation (1), zero initial condition (2), and non-zero boundary conditions (3)-(7)).

Thus, the function $c_{1}(x, t)$, which is obtained by summing the right parts of formulas (90) (at $j=1$ ) and (91), describes the desired dynamics of the concentration of metal substances in the first layer of a two-layer peat block, the function $c_{2}(x, t)$, which is obtained by summing the right parts of formulas (90) (at $j=2$ ) and (92) describes the desired dynamics of the concentration of metal substances in the second layer of a two-layer peat block. The construction of the analytical solution of the problem (1)-(8) is entirely completed.

\section{CONCLUSION}

In this paper, it is studied the problem of determining the dynamics of the concentration of metal substances in a two-layer anisotropic peat block. The work examines in detail the well-known variables separation method for constructing an analytical solution for a mathematical model of the studied problem. It is shown that the main difficulty is only the solution of interrelated auxiliary problems AP1 and AP2, which are obtained from the original mathematical model under the conditions that there are no sources in both layers, and that all boundary conditions are homogeneous

\section{REFERENCES}

[1] E. Teirumnieka, I. Kangro, E. Teirumnieks, and H. Kalis, "The analytical solution of the 3D model with Robin's boundary conditions for 2 peat layers," The 10th International Scientific and Practical Conference "Environment. Technology. Resources", vol. III, June 18-20, 2015, Rezekne, Latvia, pp. 186-192. |Online|. Available: http://journals.ru.lv/index.php/ETR/article/view/618/714. |Accessed: March 15, 2017.

[2] I. Kangro, H. Kalis, A. Gedroics, E. Teirumnieka, and E. Teirumnieks, "On mathematical modelling of metals distributions in peat layers," Journal of Mathematical Modelling and Analysis, vol. 19, no. 4, pp. 568-588, 2014.

[3] E. Teirumnieka, E. Teirumnieks, I. Kangro, H. Kalis, and A. Gedroics, "The mathematical modelling of $\mathrm{Ca}$ and Fe distribution in peat layers," The 8th International Scientific and Practical Conference "Environment. Technology. Resources", vol. II, June 20-22, 2011, Rezekne, Latvia, pp. 40-47, 2011.

[4] H. Orru and M. Orru, "Sources and distribution of trace elements in Estonian peat," Journal of Global and Planetary Change, vol. 53, no. 4, pp. 249-258, 2006.

[5] P. A. Brown, S. A. Gill, and S. J. Allen, "Metal removal from wastewater using peat," Journal of Water Research, vol. 34, no. 16, pp. 3907-3916, 2000.

[6] A. N. Tikhonov and A. A. Samarsky, Equations of Mathematical Physics. New York: Dover Publications, 1990, xvi+765 p.

[7] A. N. Tikhonov and V. Ya. Arsenin, Solution of Ill-posed Problems. Washington: Winston \& Sons, 1977, xiii+258 p.

[8] S. A. Andreyev and Sh. E. Guseynov, Regularizing algorithms for diagnosing: Applied to gas turbine engines in operation. Monograph. Saarbrücken: LAP Publishing, 2013, 116 p.

[9] M. A. Al-Gwaiz, Sturm-Liouville Theory and its Applications. London: Springer-Verlag, 2008, $\mathrm{x}+264$ p.

[10] B. M. Levitan and I. S. Sargsyan, Sturm-Liouville and Dirac Operators. Dordrecht: Kluwer Academic Publications, 1991, xi+350 p.

[11] V. A. Steklov, Fundamental Problems of Mathematical Physics. Moscow: Science, 1983, $432 \mathrm{p}$.

[12] V. A. Il'in and E. G. Poznyak, Fundamentals of Mathematical Analysis, Part II. Moscow: Science, 1980, 448 p.

[13] A. A. Abrikosov, L. P. Gor'kov, and I. E. Dzyaloshinsky, Methods of Quantum Field Theory in Statistical Physics. Englewood Cliffs: Prentice-Hall, 1963, xv+352 p.

[14] L. S. Levitov and A. V. Shitov, Green's Functions: Problems with Solutions. Moscow: FizMatLit, 2002, 252 p.

[15] V. M. Babich, V. B. Kapilevich, S. G. Mikhlin, G. I. Natanson, P. M. Riz, L. N. Slobodetsky, and M. M. Smirnov, Linear Equation of Mathematical Physics. Moscow: Science, 1964, 368 p. 